Title : will be set by the publisher

Editors : will be set by the publisher

EAS Publications Series, Vol. ?, 2009

\title{
PAST SINGULARITIES IN PHANTOM THEORIES
}

\author{
Leonardo Fernández-Jambrina ${ }^{1}$
}

\begin{abstract}
FLRW models filled with just dark energy are shown to have a finite past, since causal geodesics cannot be extended beyond a certain proper time. It is shown that curvature measured along causal geodesics becomes infinity on travelling to the past, though curvature scalars tend to zero. Furthermore the time measured by free-falling observers from coincidence time to Big Rip is shown to be as short as wished by increasing their linear momentum.
\end{abstract}

\section{Introduction}

The analysis of several sources of observational data, supernovae type Ia, redshift of distant objects, temperature fluctuations of background radiation (cfr. Padmanabhan 2005 for a nice review) has lead to accept that our universe is acceleratedly expanding nowadays.

This unexpected feature, which cannot be explained by means of an standard matter content for the universe, has motivated lots of theoretical work, such as a dark energy component in the energy-momentum tensor or modifications of the theory of gravitation. Violation of energy conditions due to these new components have given rise to consider new types of singularities, different from the wellknown Big Bang and Big Crunch. Beyond the so-called phantom divide, $w<-1$, accelerated expansion ends up at a Big Rip (Caldwell 2002). Below the phantom divide, other atypical events may come up, such as sudden singularities (Barrow 2004).

Usually the analysis of these singularities is made by just tracking where the curvature scalars blow up. For instance, a classification of singularities is provided (Nojiri and Odintsov 2005) depending on which scalars are singular or not. However the use of more poweful tools, such as geodesic analysis, has proven quite

\footnotetext{
1 E.T.S.I. Navales

Universidad Politécnica de Madrid

Arco de la Victoria s/n

28040-Madrid, Spain
} 
Title : will be set by the publisher

successful to discover new features of the singularities. Furthermore, this is the natural framework to talk about singularities (Hawking and Ellis 1973).

\section{FLRW models}

We consider homogeneous and isotropic universes endowed with a FriedmannLemaître-Robertson-Walker metric

$$
d s^{2}=-d t^{2}+a^{2}(t)\left\{f^{2}(r) d r^{2}+r^{2} d \Omega^{2}\right\}, \quad f^{2}(r)=\frac{1}{1-k r^{2}}, \quad k=0, \pm 1,
$$

where the coordinates have the usual ranges. We are mostly interested in spatiallyflat models, $k=0$, since observations point out this kind of universe.

Geodesics in these models are described by simple ordinary equations due to their high symmetry,

$$
\begin{aligned}
\dot{t} & =\sqrt{\delta+\frac{P^{2}}{a^{2}(t)}}, \\
\dot{r} & = \pm \frac{P}{a^{2}(t) f(r)} .
\end{aligned}
$$

The dot stands for derivation with respect to proper time $\tau$, which allows parametrization of geodesics satisfying the unitary condition of the velocity,

$$
\delta=\dot{t}^{2}-a^{2}(t) f^{2}(r) \dot{r}^{2},
$$

where $\delta$ is one for timelike, zero for lightlike or minus one for spacelike geodesics. The constant $P$ is the specific linear momentum of the geodesic.

There are then three families of causal geodesics: lightlike geodesics $(\delta=0)$, fluid worldlines $(\delta=1, P=0)$ and timelike radial geodesics $(\delta=1, P \neq 0)$.

In order to perform calculations with a general scale factor, we only need that $a(t)$ has a power expansion (Cattoën and Visser 2005) around the event at $t_{0}$, where a singularity is deemed to appear,

$$
a(t)=c_{0}\left|t-t_{0}\right|^{\eta_{0}}+c_{1}\left|t-t_{1}\right|^{\eta_{1}}+\cdots,
$$

with real and ordered exponents, $\eta_{0}<\eta_{1} \cdots$, and positive $c_{0}$. Hence, the model behaves close to $t_{0}$ as a power-law model of exponent $\eta_{0}$. Accordingly, several types of singular events may arise:

- $\eta_{0}>0$ : the scale factor vanishes at $t_{0}$ and we have either a Big Bang or Big Crunch.

- $\eta_{0}=0$ : the scale factor is finite at $t_{0}$. If $a(t)$ is analytical, the event at $t_{0}$ is regular. Otherwise a weak or sudden singularity appears.

- $\eta_{0}<0$ : the scale factor diverges at $t_{0}$ and a Big Rip appears. 


\begin{tabular}{|c|c|c|c|c|c|}
\hline$\eta_{0}$ & $\eta_{1}$ & $k$ & $c_{0}$ & Tipler & Królak \\
\hline$(-\infty, 0)$ & $\overline{\left(\eta_{0}, \infty\right)}$ & \multirow{4}{*}{$0, \pm 1$} & \multirow{5}{*}{$(0, \infty)$} & Strong & Strong \\
\hline \multirow[t]{2}{*}{0} & $(0,1)$ & & & Weak & Strong \\
\hline & {$[1, \infty)$} & & & Weak & Weak \\
\hline$(0,1)$ & $\left(\eta_{0}, \infty\right)$ & & & Strong & Strong \\
\hline \multirow[t]{4}{*}{1} & $(1, \infty)$ & 0,1 & & Strong & Strong \\
\hline & $(1, \infty)$ & \multirow{3}{*}{-1} & $(0,1) \cup(1, \infty)$ & Strong & Strong \\
\hline & $(1,3)$ & & \multirow[t]{2}{*}{1} & Weak & Strong \\
\hline & {$[3, \infty)$} & & & Weak & Weak \\
\hline$(1, \infty)$ & $\left(\eta_{0}, \infty\right)$ & $0, \pm 1$ & $(0, \infty)$ & Strong & Strong \\
\hline
\end{tabular}

Table 1. Singularities in FLRW models

The analysis of causal geodesics is easy to perform in this case and is shown in Table 1 (Fernández-Jambrina and Lazkoz 2006).

This table shows the first of the unusual features of sudden singularities (Fernández-Jambrina and Lazkoz 2004): they are weak according to Tipler (Tipler 1977) and Królak's definitions (Krolak 1986) and therefore they do not exert infinite tidal forces that might destroy extended bodies going through them. In this sense, they cannot be considered the final fate of a universe, since they can be avoided by some observers.

Concerning the Big Rip, another issue unveiled by the survey of causal geodesics in FLRW models with an effective equation of state $p=w \rho, w \in(-5 / 3,-1)$ for late times is that lightlike geodesics need infinite proper time before meeting the Big Rip. That is, only massive objects see the Big Rip. In this sense, since photons do not reach this event, we could say that Big Rip may be considered quite an intimate experience... with lights turned out! Since dark energy models with $w$ just below minus one have been shown to be compatible with observations, this features cannot be neglected as mathematical curiosities.

\section{What about an infinite $t_{0}$ ?}

The issue of singularities located at infinite time coordinate is not as pointless as it may seem at first glance. The example of photons close to a Big Rip shows us that a finite coordinate time lapse can be experienced as an infinite proper time lapse, in this case by massless particles. The relation between proper time and coordinate time is far from trivial.

First, we have to determine when causal geodesics reach infinite coordinate time in finite proper time.

For lightlike geodesics we may integrate the geodesic equation (2.1),

$$
\dot{t}=\frac{P}{a(t)} \Rightarrow \int_{t_{0}}^{t} a\left(t^{\prime}\right) d t^{\prime}=P\left(\tau-\tau_{0}\right)
$$


Title : will be set by the publisher

in order to attain a necessary and sufficient condition for reaching infinite coordinate time in finite proper time: integrability of the scale factor $a(t)$,

$$
\int_{t}^{\infty} a\left(t^{\prime}\right) d t^{\prime}<\infty
$$

for sufficiently large $t$.

The same condition is valid for radial timelike geodesics. On the contrary, fluid worldlines with $P=0$ are not to be considered since for them proper time is essentially coordinate time.

For a start, we may consider models which behave for large $t$ as a power-law model $a(t) \simeq c t^{\eta}$. For them the geodesic equation (2.1) yields

$$
t \simeq \begin{cases}\left\{\frac{(1+\eta) P}{c}\right\}^{1 /(1+\eta)}\left(\tau-\tau_{0}\right)^{1 /(1+\eta)} & \eta \neq-1, \\ e^{P\left(\tau-\tau_{0}\right) / c} & \eta=-1,\end{cases}
$$

and obviously infinite $t$ is reached for finite $\tau_{0}$ if and only if $\eta<-1$.

This is not however the interesting case, since it involves models starting with a Big Rip at $t=0$. But if we consider models starting at $t=-\infty$ and ending up at a Big Rip at $t=0$ the same reasoning is valid. The origin of radial causal geodesics would be at a finite proper time.

At this point, two possibilities arise in order to explain the finite origin of causal geodesics in phantom models: there is either a singularity at $t=-\infty$ which explains the abrupt startpoint of these geodesics instead of ranging from and infinite proper time or the FLRW coordinate patch does not cover the whole universe and we need to enlarge it.

This is done, for instance, with de Sitter universes in the parametrization which is used for inflation, $k=0, a(t)=e^{\sqrt{\Lambda / 3}}$, which fulfills condition (3.1) and therefore its radial geodesics reach $t=-\infty$ in finite proper time. However, in this case it is possible to extend the spacetime to a larger one, $k=1$, $a(T)=\sqrt{3 / \Lambda} \cosh (\sqrt{\Lambda / 3} T)$ with another change of coordinates (Hawking and Ellis 1973) and hence the singularity at $t=-\infty$ is only apparent and due to a bad choice of coordinates.

This option appears to be the correct for other universes since at $t=-\infty$ all curvature scalars vanish as $1 / t^{2}$ for power-law models.

However if we reckon Ricci tensor components along radial lightlike geodesics, unexpected features arise,

$$
u^{t}=\frac{P}{a}, \quad u^{r}= \pm \frac{P}{f a^{2}}, \quad R_{i j} u^{i} u^{j}=2 P^{2}\left(\frac{a^{\prime 2}+k}{a^{4}}-\frac{a^{\prime \prime}}{a^{3}}\right) \simeq \frac{2 P^{2} \eta}{c^{2} t^{2(\eta+1)}}+\frac{2 k P^{2}}{c^{4} t^{4 \eta}},
$$

since the first term diverges at $t=-\infty$ for $\eta<-1$ regardless of the value of $k$. The same happens for radial timelike geodesics. 
This unexpected result could only be avoided in models for which the curvature term cancels the first term, $a^{2}+k=a a^{\prime \prime}$. But curiously the solutions of this equation are well known universes.

For $k=0$ we recover the aforementioned parametrization of de Sitter universe, $a(t)=e^{\sqrt{\Lambda / 3} t}$. For $k=1$ we find another parametrization of de Sitter universe, $a(t)=\sqrt{3 / \Lambda} \cosh (\sqrt{\Lambda / 3} t)$. And for $k=-1$, also another parametrization of de Sitter spacetime arises, $a(t)=\sqrt{3 / \Lambda} \sinh (\sqrt{\Lambda / 3} t)$ but also anti-de Sitter spacetime, $a(t)=\sqrt{3 / \Lambda} \cos (\sqrt{\Lambda / 3} t)$, but for a choice of time coordinate origin. Since only the one with $k=0$ decreases at $t= \pm \infty$, they do not affect our results.

Therefore we are led to conclude that universes which asymptotically behave as power-law models with $\eta<-1, w \in(-5 / 3,-1)$, in spite of having vanishing curvature scalars at $t=-\infty$, they possess there a sort of directional singularity that affects only radial geodesics, a p.p. curvature singularity (curvature singularity along a parallelly transported basis) (Hawking and Ellis 1973). Furthermore, this singularity is strong (Tipler 1977, Królak 1986) and therefore cannot be overlooked.

Radial observers would start their trajectories at a singularity at $t=-\infty$ and would end up at a Big Rip at $t=0$, though their life span would be finite, as we have shown. Photons would start their paths at the singularity at $t=-\infty$ but they would never reach the Big Rip in these models.

Summarizing these results and extending the conclusions to non-asymptotically power-law models, we have:

- For scale factors growing or decreasing as $1 /|t|$ or slower, radial causal geodesics reach $t=-\infty$ in infinite proper time.

- For scale factors decreasing faster than $1 /|t|$, radial causal geodesics reach $t=-\infty$ in finite proper time, meeting a strong curvature singularity, except for de Sitter spacetime.

Cases with an ill-defined limit at $t=-\infty$ may be handled directly with condition (3.1).

\section{Duration of the universe}

The features which have been shown about the past of phantom models might seem useless since dark energy is a relevant component only for the future of the universe from coincidence time on in order to explain accelerated expansion, not for the past. Nonetheless there are implications for the models.

For instance, we may use our results to reckon the duration of a universe filled with a power-law phantom field as measured by an observer following a radial geodesic (2.1),

$$
T=\int_{-\infty}^{0} \frac{d t}{\sqrt{1+P^{2} / a^{2}(t)}}=\int_{-\infty}^{0} \frac{d t}{\sqrt{1+P^{2} / c^{2} t^{2 \eta}}}
$$




$$
=\left(\frac{P}{c}\right)^{1 / \eta} \int_{0}^{\infty} \frac{x^{\eta} d x}{\sqrt{1+x^{2 \eta}}}=\left(\frac{P}{c}\right)^{1 / \eta} I,
$$

which is finite again for $\eta<-1$.

But most surprising is that this quantity can be made as small as wanted by increasing the total linear momentum $P$ of the traveller. It may be argued that this calculation is useless since we cannot extend the validity of the phantom model towards the past, but this does not invalidates the result. We may calculate the time span measured by the observer from coincidence time to the Big Rip and reach the same conclusion: observers may reduce the life of the universe by increasing their linear momentum. We have always known that driving fast could be dangerous, but not to this extent! Since a negative exponent $\eta$ is necessary in (4.1) for this result, it is clear that this behaviour is exclusive of phantom models with $w<-1$ for large times, that is, below the phantom divide.

So far we have shown that phantom fields produce not only a Big Rip but a directional singularity in the past, that massless particles do not experience a Big Rip and that the duration of the universe may be shortened ad libitum by increasing the linear momentum of the observer. There are other exotic features inherent to phantom models (Fernández-Jambrina 2007), especially of those which are closer to the phantom divide, but these should be enough to show the unexpected behaviour that one encounters on trying to model dark energy with phantom fields.

\section{Acknowledgments}

This work is supported by the Spanish Ministry of Education and Science research grant FIS-2005-05198. The author wishes to thank R. Lazkoz, J.M.M. Senovilla

and R. Vera for valuable discussions and the University of the Basque Country for their hospitality. The author also wants to thank the organizers of the 2007 Spanish Relativity Meeting for a superb conference.

\section{References}

Padmanabhan, T. 2005, Curr. Sci., 88, 1057.

Caldwell, R.R, 2002, Phys. Lett. B. 545, 23.

Barrow, J.D. 2004, Class. Quant. Grav. 21, L79; Nojiri, S., Odintsov, S.D. 2004, Phys. Lett. B 595, 1; Barrow J.D. 2004 Class. Quant. Grav. 21, 5619; Lake K. 2004, Class. Quant. Grav. 21, L129; Nojiri, S., Odintsov, S.D., 2004, Phys. Rev. D 70, 103522; Dąbrowski, M.P. 2005, Phys. Rev. D 71, 103505; Barrow, J.D., Tsagas, C.G. 2005 Class. Quant. Grav. 22, 1563 ; Chimento, L.P., Lazkoz, R. 2004, Mod. Phys. Lett. A 19, 2479; Dạbrowski, M.P., Phys. Lett. B 625, 184; Balcerzak, A., Dạbrowski M.P. 2006, Phys. Rev. D 73, 101301.

Nojiri, S., Odintsov, S.D., Tsujikawa, S., 2005, Phys. Rev. D, 71, 063004.

Hawking, S.W, Ellis, G.F.R., The Large Scale Structure of Space-time, Cambridge University Press (Cambridge). 
Cattoën, C. Visser, M., 2005, Class. Quant. Grav., 22, 4913.

Fernández-Jambrina, L., Lazkoz, R., 2006, Phys. Rev. D, 74, 064030.

Fernández-Jambrina, L., Lazkoz, R., 2004, Phys. Rev. D, 70, 121503(R).

Tipler, F.J., 1977, Phys. Lett. A, 64, 8.

Królak, A., 1986, Class. Quant. Grav. 3, 267.

Fernández-Jambrina, L., 2007, Phys. Lett. B, 656, 9. 